\title{
Patients' Characteristics Effecting the Use of a MyChart Patient Portal
}

\author{
Ali Ahmed \\ Manning School of Business \\ University of Massachusetts Lowell \\ Ali_Ahmed@student.uml.edu \\ Abdullah Wahbeh \\ Slippery Rock University of Pennsylvania \\ Abdullah.Wahbeh@sru.edu
}

\author{
Tareq Nasralah \\ Dakota State University \\ Tareq.Nasralah@,dsu.edu \\ Cherie B. Noteboom \\ Dakota State University \\ Cherie.Noteboom@dsu.edu
}

\begin{abstract}
In this study, we have studied the effects of patients' characteristics on the use of an online patient portal. We have created an explanatory model to illustrate the relationship between the patient-portal use and the patients' demographic and health characteristics. The study is conducted on a large dataset of 1 million patient records, provided by a leading national healthcare provider. Our results indicate, that a unit increase in health problems increases the odds of using the patient portal by $31.1 \%$. Moreover, a single visit to an emergency department decreases the odds of using a patient portal by $7.1 \%$, while a visit to an urgent care center decreases the odds of portal use by $9.2 \%$. We also found that female patients are $58 \%$ more likely to use the patient portal as compared to the male patients. The study has practical implications for health care providers, patients and portal developers.
\end{abstract}

\section{Introduction}

In the domain of healthcare delivery, patients' access to their health records has the potential to improve their health outcomes $[3,26]$. Providing personal health records to the patients establishes a more collaborative relationship between caregivers and patients $[9,23]$. To increase this collaboration, healthcare organizations have developed applications which electronically communicate between providers and patients. These applications are called patient portals, which are group of online applications developed to allow patients to securely access their health information with a potential to improve care quality and reduced associated cost [14, 16]. Patient portals have the potential to bring together providers and patients to mediate care outside of traditional healthcare centers and clinics $[6,12,24]$. Moreover, patient's engagement has been identified as one of the pillars in the healthcare provisions, for reducing the healthcare cost and improving the quality of US healthcare [20]. Even though many hospitals have implemented patient portals, the adoption and use of these portals by patients is still very low [2]. The goal of this research is to gain insight of patients' characteristics, which are significantly affecting the use and adoption of these portals.

In this research, we have used a large dataset to develop a relationship for patients' demographic and health characteristics that are affecting the use of the MyChart patient portal. We have created an explanatory model, to estimate this relationship. Specifically, we have analyzed the effect of patient's age, gender, race, ethnicity, insurance type, health problems, emergency department visits, and urgent care visits, on the use of the patient portal. The aim was to find the magnitude, direction and significance of these factors affecting the use of the patient portal. The patient portal under study is a MyChart patient portal, developed by EPIC Healthcare Software ${ }^{1}$. According to EPIC, the company currently holds medical records of $54 \%$ of all the patients in the United States and $2.5 \%$ of all the patients worldwide [10].

\section{Background}

Patient portals are an effective healthcare communication technology that benefits both, patients and healthcare providers. The benefits of patient portals for healthcare providers include improved providerpatient communication, decrease in recordkeeping costs, decrease in repeated lab tests, shorter hospital stays, higher patient safety rates, and reduced medical errors [7]. Patient portal is a provider-tethered application that provides patients with online digital access to health-

\footnotetext{
${ }^{1}$ https://www.epic.com
} 
care services and information provided directly by an ambulatory-care $[5,8]$. In addition to providing access to personal medical records, patient portals provide patients with virtual care, such as the ability to schedule an electronic visit via the portal, and real-time video visits where patients interact with a healthcare provider instead of traveling to the clinic or hospital $[8,19]$.

It is important for providers and hospitals to understand the patient characteristics that are significantly affecting the use of patient portal $[4,6]$. This understanding can improve the adoption and use of patient portals and also helps in fulfilling the healthcare needs of patients [24]. Understanding the factors affecting the utilization of patient portals is still an underexplored research area. In the literature, studies which evaluated patient characteristics associated with the use of patient portals found that portal users were more likely to be female, middle-aged, with much greater level of illness than non-users [25, 27]. The University of Pittsburgh (Pittsburgh, Pennsylvania) evaluated characteristics of early adopters of patient portals [15]. They have analyzed the patients' characteristics who have utilized the E-visit functionality of a patient portal. The study found that E-visits users were more likely to be female, young, not retired, and in poorer health condition, as indicated by greater numbers of diagnoses and medications [15]. In another study conducted by the Cleveland Clinic, Department of Internal and Family Medicine, found that portal users were more likely to be young, have commercial insurance, and belong to a White race group [28]. Similarly, Osborn et al., (2013), found that portal users are likely to be Caucasian/White, have higher incomes, and have private insurance [24]. The most common portal actions were found to be viewing test results (37\%), viewing and responding to clinic messages (29\%), and sending medical advice requests (56\%) [8]. Another empirical study found that $58 \%$ of patient portal users were White, and all other racial/ethnic minority groups had poorer baseline adherence statistic as compared to Whites [19]. Other major factors studied in previous research studies include, diagnosis (symptoms and severity) $[13,17,18,21,22]$, age $[13,17,18]$, limited knowledge / education / health literacy [13, 21], limited computer and internet access [21], socioeconomic condition, Medicaid recipients [17], ethnicity[13, 17], attitude, and trust [19].

Our research is different from previous research studies in multiple ways. First, most of the previous studies were conducted on a small dataset and used methodologies such as observational cohort study [18], qualitative semi-structured interviews [21], crosssectional analysis [17], empirical evaluation [22], or literatures reviews [13]. To the best of our knowledge, none of the previous studies have used a large dataset of over 1 million patient records to empirically measure the effect of key patient's characteristics on the use and adoption of a patient portal. Second, we are using two new patient's characteristics which may influence the use of patient portal. These are Emergency Department (ED) visits and Urgent Care Center (UCC) visits. Previously these characteristics have been studied in a slightly different manner. The $[1,6,11]$ have measured how the patient portal usage increases or decrease the visit to an emergency department or urgent care center. Whereas, we are analyzing this relationship in an inverse manner, i.e. analyzing the effect of Emergency Department (ED) and Urgent Care Center (UCC) visits on the use of the patient portal. We are trying to answer the questions, whether the use of ED or UCC visit increase or decrease the chances of accessing the patient portal. To the best of our knowledge no one has previously explored this relationship in this way while using a large dataset.

\section{Data \& Methodology}

In this study, we have examined the patients' demographic and health characteristics and its effects on the adoption and utilization of a patient portal. The dataset used in this study is a real-world patient dataset, provided by a leading private healthcare system in the nation. It was a limited dataset that was stripped-off protected health information (PHI), reviewed by a privacy board and received the IRB approval. The dataset contained 1,142,691 randomly sampled patient records. Each record contains patient's demographic characteristic, health characteristics, and portal utilization information for the last three years. The demographics variables are age, gender, race, ethnicity and insurance type. The class distribution of all the demographic characteristics are presented in Table 1.

Table 1. Distribution of Demographic Characteristic

\begin{tabular}{|l|l|l|}
\hline Gender & 597,793 & $52.29 \%$ \\
\hline Male & 545,235 & $47.70 \%$ \\
\hline Female & $\mathbf{1 , 1 4 3 , 0 2 8}$ & $\mathbf{1 0 0 \%}$ \\
\hline \multicolumn{3}{|l|}{} \\
\hline Age & 228,247 & $19.97 \%$ \\
\hline $0-14$ & 230,998 & $20.21 \%$ \\
\hline $15-29$ & 208,275 & $18.22 \%$ \\
\hline $30-44$ & 202,386 & $17.71 \%$ \\
\hline $45-59$ & 173,784 & $15.20 \%$ \\
\hline $60-74$ & 99,338 & $8.69 \%$ \\
\hline $75+$ & $\mathbf{1 , 1 4 3 , 0 2 8}$ & $\mathbf{1 0 0} \%$ \\
\hline \multicolumn{3}{|l|}{} \\
\hline Race & $1,003,080$ & $87.76 \%$ \\
\hline White & 28,677 & $2.51 \%$ \\
\hline Black or African American & 49,026 & $4.29 \%$ \\
\hline American Indian/Alaska Native
\end{tabular}




\begin{tabular}{|l|l|l|}
\hline Asian & 13,296 & $1.16 \%$ \\
\hline $\begin{array}{l}\text { Native Hawaiian/Pacific } \\
\text { Islander }\end{array}$ & 1,792 & $0.16 \%$ \\
\hline Others & 47,157 & $4.13 \%$ \\
\hline \multicolumn{3}{|l|}{} \\
\hline Ethnic Group & $\mathbf{1 4 3 , 0 2 8}$ & $\mathbf{1 0 0 \%}$ \\
\hline Not Hispanic or Latino & $1,071,283$ & $93.72 \%$ \\
\hline Hispanic or Latino & 35,390 & $3.10 \%$ \\
\hline Others & 36,355 & $3.18 \%$ \\
\hline \multicolumn{2}{|l|}{$\mid$} \\
\hline Insurance Company & $\mathbf{1 , 1 3 , 0 2 8}$ & $\mathbf{1 0 0 \%}$ \\
\hline Medicare & 199,286 & $17.43 \%$ \\
\hline Medicaid & 141,947 & $12.42 \%$ \\
\hline $\begin{array}{l}\text { BCBS (Blue Cross Blue } \\
\text { Shield) }\end{array}$ & 401,965 & $35.17 \%$ \\
\hline Other Private Insurance & 399,830 & $34.98 \%$ \\
\hline & $\mathbf{1 , 1 4 3 , 0 2 8}$ & $\mathbf{1 0 0 \%}$ \\
\hline
\end{tabular}

The patient health characteristics include the variables for the number of health problems a patient has, the number of times a patient has visited an emergency department in the last three years and the number of times a patient has visited an urgent care center in the last three years. The Table 2 provides descriptive statistics for all these health characteristics.

Table 2. Descriptive Statistics of Health Characteristics

\begin{tabular}{|l|c|}
\hline \multicolumn{2}{|l|}{ Number of Health Problems } \\
\hline Mean & 0.59 \\
\hline Standard Deviation & 1.40 \\
\hline Minimum & 0.0 \\
\hline Maximum & 29.0 \\
\hline Number of Emergency Department Visits (ED visits) \\
\hline Mean & 0.523 \\
\hline Standard Deviation & 1.2 \\
\hline Minimum & 0.0 \\
\hline Maximum & 167 \\
\hline Number of Urgent Care Visits (UCC Visits) \\
\hline Mean & 0.687 \\
\hline Standard Deviation & 1.89 \\
\hline Minimum & 0.0 \\
\hline Maximum & 68.0 \\
\hline
\end{tabular}

The portal utilization information is represented by a categorical variable with two classes; Accessed $=1$; representing if a patient has accessed the portal in the last three years and Accessed $=0$; representing otherwise. Table 3 provides the class distribution of the variable Portal Accessed.
Table 3. Portal Access Distribution

\begin{tabular}{|l|l|l|}
\hline \multicolumn{3}{|l|}{ Patient Portal Accessed } \\
\hline Accessed $=0$ & 833,383 & $72.91 \%$ \\
\hline Accessed $=1$ & 309,645 & 27.08 \\
\hline & $1,143,028$ & $100 \%$ \\
\hline
\end{tabular}

To analyze the factors effecting the use and adoption of the patient portal, we perform a multivariate logistic regression analysis as it is suitable for the binary dependent variable. The dependent variable in our regression model is whether a patient has accessed the portal in last three years or not (i.e. Portal Accessed). The following equation represents our explanatory model with all the categorical and continuous variables.

$$
\begin{aligned}
\operatorname{Pr}(\text { Accessed }=1) & =G(\text { Constant } \\
& +\beta_{1} \text { HealthProblems } \\
& +\beta_{2} \text { EDVisits }+\beta_{3} \text { UCCVisits } \\
& +\beta_{4} \text { Female }+\beta_{5 i} \text { Age }_{i}+\beta_{6 j} \text { Race }_{j} \\
& +\beta_{7 k} \text { Ethinicity }_{k}+\beta_{8 l} \text { Insurance }_{l} \\
& \left.+\beta_{9} \text { HealthProblems }+\varepsilon\right)
\end{aligned}
$$

where $G\left(\beta^{\prime} \boldsymbol{X}\right)=e^{\beta^{\prime} X}\left(1+e^{\beta^{\prime} X}\right)$

and

$i=$ Age categories $1-5$

$j=$ Race categories $1-6$,

$k=$ Ethinicity categorires $1-2$

$l=$ insurance categories $1-3$

\section{Results and Discussion}

In this study, we have explored the relationship between patient's demographic \& health characteristics and patient portal use. Our explanatory model specifically explored the effects of patient's age, gender, race, ethnicity, insurance type, health problems, EDvisits and UCC-visits. The results of our model are shown in Table 4 . We found consistently significant (pvalue $<0.01)$ relationship for most of the independent variables.

We have estimated that a one-unit increase in health problems increases the odds of using the patient portal by $31.1 \%$. A single visit to an emergency department decreases the odds of using a patient portal by $7.1 \%$, while a visit to an urgent care center decreases the odds of portal use by $9.2 \%$.

In the terms of demographic characteristics, we have estimated that the odds of using a patient portal by a female user is $58 \%$ higher as compared to the odds of a male user. Similarly, some age groups have higher odds 
of using the patient portal compared to others. The base age group in the model is the group of $0-14$. The users of this age group are most likely to be parents of the children who are between age group $0-14$ and registered in the patient portal. The odds of using the patient portal by the age group of $30-44$ are the highest as compared to all other age groups, they are almost 10.12 times more likely to use the patient portal compared to the parents who are using on behalf of their children. Moreover, the estimated odds of using the patient portal decreases after the age of 59, while the odds of using a patient portal for the age group of 75 and above is least compared to other age groups. These findings are consistent with the findings of Palen et al. [25] and Ralston et al. [27]. The effect of age group 6074 was not consistent and found insignificant in the model.

Table 4. Logistic Regression Results

\begin{tabular}{|c|c|c|c|}
\hline \multicolumn{4}{|l|}{ Logistic Regression Model } \\
\hline \multicolumn{4}{|c|}{ Explaining Patient Portal Access. DV: Accessed $=1$} \\
\hline Variables & Coeff. & $\begin{array}{l}\text { Std. } \\
\text { Err. }\end{array}$ & $\begin{array}{l}\text { Odds } \\
\text { Ratio } \\
\end{array}$ \\
\hline Constant & $-0.0293 * *$ & 0.212 & \\
\hline \multicolumn{4}{|l|}{ Health Characteristics } \\
\hline Health Problems & $0.271 * * *$ & 0.004 & 1.311 \\
\hline ED Visits & $-0.073^{* * *}$ & 0.003 & 0.929 \\
\hline UCC Visits & $-0.096 * * *$ & 0.007 & 0.908 \\
\hline \multicolumn{4}{|l|}{ Gender } \\
\hline Female & $0.458 * * *$ & 0.062 & 1.580 \\
\hline \multicolumn{4}{|l|}{ Age Groups } \\
\hline $15-29$ & $1.523 * * *$ & 0.310 & 4.585 \\
\hline $30-44$ & $2.315 * * *$ & 0.232 & 10.12 \\
\hline $45-59$ & $0.121 * *$ & 0.621 & 1.128 \\
\hline $60-74$ & $-0.030-$ & 0.023 & 0.970 \\
\hline $75+$ & $-0.843^{*}$ & 0.426 & 0.430 \\
\hline \multicolumn{4}{|l|}{ Race } \\
\hline Black or African American & $-1.394 * * *$ & 0.026 & 0.248 \\
\hline American Indian/Alaska Native & $-1.782 * * *$ & 0.022 & 0.168 \\
\hline Asian & $-0.485^{*}$ & 0.032 & 0.615 \\
\hline Native Hawaiian/Pacific Islander & $-1.146^{* *}$ & 0.103 & 0.032 \\
\hline Others & $-0.473 * * *$ & 0.043 & 0.623 \\
\hline \multicolumn{4}{|l|}{ Ethnic Group } \\
\hline Hispanic or Latino & $-0.718 * * *$ & 0.048 & 0.487 \\
\hline Others & -0.110 & 0.022 & 0.865 \\
\hline \multicolumn{4}{|l|}{ Insurance Company } \\
\hline Medicare & $-0.204 * * *$ & 0.010 & 0.815 \\
\hline Medicaid & $-0.403 * * *$ & 0.008 & 0.668 \\
\hline BCBS & $0.106^{* * * *}$ & 0.005 & 1.111 \\
\hline No. Observations & \multicolumn{3}{|l|}{$1,143,028$} \\
\hline Degrees of Freedom & \multicolumn{3}{|l|}{18} \\
\hline DF Residual & \multicolumn{3}{|l|}{$1,143,009$} \\
\hline $\mathrm{p}>\chi^{2}$ & \multicolumn{3}{|l|}{$<0.01 * * *$} \\
\hline Pseudo R2 & \multicolumn{3}{|l|}{0.099} \\
\hline Model / Method & \multicolumn{3}{|c|}{ Logit / MLE } \\
\hline
\end{tabular}

The White race is the base group for the race category. All the other races are estimated to be less likely to use the patient portal as compared to the White race. The Native Hawaiian \& Pacific Islander race patients has the least odds of using the patient portal. They are $96.8 \%$ less likely to use the patient portal compared to the White race patients. This is also consistent with previous research studies [15, 25]. Similarly, the odds of using the portal with Hispanics or Latino ethnicity are 0.487 times less compared to NonHispanics.

Our model has estimated a negative relationship between portal use and the Medicaid/Medicare insurance type. The patients with the private insurance companies, such as Blue Cross Blue Shield, have a positive relationship with the use of the patient portal. The Medicare patients are $18.5 \%$ less likely to use the patient portal compared to a private insurance company. Similarly, the odds of using the patient portal by a patient having Medicaid insurance is about 33\% less compared to the odds of patients who have a private or commercial insurance. These findings are also consistent with the finding of $[10,11]$, who found that private insurance buyers are more likely to use the patient portal. The estimated Pseudo $\mathrm{R}^{2}$ for model is 0.09 , it is calculated using McFadden's statistics, which uses a ratio of the log likelihood of the intercept model and the log likelihood of the full model. This shows that the fitted model is better than the non-fitted models and explains the variability to some extent.

\section{Conclusion}

In this research, we have used a large dataset of over 1 million patient records to create an explanatory model. The model has explained the relationship between patient's portal use and their demographic and health characteristics. Specifically, the model has estimated the effect of age, gender race, ethnicity, insurance type, health problems, ED visits, and UCC visits on patient's portal use. Many of the findings were consistent with previous research findings. Such as, the use of patient portal of females, young \& middle aged, whites and patients having commercial insurance are more likely to use the patient portal. The two new factors used in this research are the Emergency department (ED) visits and Urgent care centers (UCC) visits. We have found a negative relationship between EDs and UCCs visits and the use of the patient portal.

In this research, we have contributed to the field of healthcare IT in three ways; first, we have used a realworld patient dataset of a large size. Most of the previous studies were conducted on small samples using surveys and other historical datasets. Second, we have confirmed many findings of the previous studies, which were conducted using small empirical studies, qualitative semi-structured interviews, cross-sectional analysis, or literature reviews methodologies. Third, we have used 
two new factors, ED visits and UCC visits, effecting the patients' use of patient portals.

There are number of limitations in the paper. First, we have not incorporated any information about patient's illness type. Patients with chronic illnesses may have higher impact on the use of patient portal. Second, the dataset contains unrealistic proportions of the race groups. For example, we have more Native and Asian race people in the dataset as compared to Blacks.

This paper is our first paper using this dataset. In our future studies, we plan to add more factors, such as patient's illness type, family history and geographic location. Future studies can also include, predictive models and machine learning techniques to estimate the predictive accuracy of these factors. We also plan to study the frequency of portal use by the patient, which can be a very interesting research question. This research has set the stage for us as well as for other researchers to use real-world large datasets to explore the adoption of IT in healthcare settings.

\section{References}

[1] Ahmed, A., T. Nasralah, Y. Wang, et al., "Impact of E-Visits on Emergency Departments and Urgent Care Centers", 2017.

[2] Al-Ramahi, M., A. Wahbeh, and C. Noteboome, "Features Related to Patient Portal User Satisfaction: NGram-Based Analysis of Users' Feedback", Proceedings of the 2018 ACM SIGMIS Conference on Computers and People Research, ACM (2018), 152.

[3] Al-Ramahi, M.A., J. Liu, and O.F. El-Gayar, "Discovering Design Principles for Health Behavioral Change Support Systems: A Text Mining Approach", ACM Trans. Manage. Inf. Syst. 8(2-3), 2017, pp. 5:1-$5: 24$.

[4] Ammenwerth, E., P. Schnell-Inderst, and A. Hoerbst, "The impact of electronic patient portals on patient care: a systematic review of controlled trials", Journal of medical Internet research 14(6), 2012.

[5] Baird, A., M.F. Furukawa, and T.S. Raghu, "Understanding contingencies associated with the early adoption of customer-facing web portals", Journal of Management Information Systems 29(2), 2012, pp. 293324.

[6] Emont, S., "Measuring the impact of patient portals", California Healthcare Foundation, 2011, pp. 1-20.

[7] Erdeljac, H.P., M.J. Snider, K. Bartsch, and R. Weiss, "Patient Portals in Pharmacist-run Ambulatory Care
Clinics: Is There 'Meaningful Use'?', Excerpts in Pharmacy Research Journal 2(1), 2016, pp. 5.

[8] Gerber, D.E., A.L. Laccetti, B. Chen, et al., "Predictors and intensity of online access to electronic medical records among patients with cancer", Journal of oncology practice 10(5), 2014, pp. e307-e312.

[9] Giardina, T.D., S. Menon, D.E. Parrish, D.F. Sittig, and H. Singh, "Patient access to medical records and healthcare outcomes: A systematic review", Journal of the American Medical Informatics Association 21, 2014, 737-741.

[10] Glaze, J., "Epic Systems draws on literature greats for its next expansion", Wisconsin State J, 2015.

[11] Goldzweig, C.L., G. Orshansky, N.M. Paige, et al., "Electronic patient portals: evidence on health outcomes, satisfaction, efficiency, and attitudes: a systematic review", Annals of internal medicine 159(10), 2013, pp. 677-687.

[12] Hogan, T.P., K.M. Nazi, T.M. Luger, et al., "Technology-assisted patient access to clinical information: An evaluation framework for blue button", Journal of Medical Internet Research 16(3), 2014.

[13] Irizarry, T., A.D. Dabbs, and C.R. Curran, "Patient portals and patient engagement: a state of the science review", Journal of medical Internet research 17(6), 2015 .

[14] Islam, M.N., M. Kabir, J. Sultana, et al., "Autism Sohayika: A Web Portal to Provide Services to Autistic Children", International Conference on Mobile Web and Intelligent Information Systems, Springer (2018), 181192.

[15] Jung, C., R. Padman, G. Shevchik, and S. Paone, "Who are portal users vs. early e-Visit adopters? A preliminary analysis", AMIA Annual Symposium Proceedings, American Medical Informatics Association (2011), 1070.

[16] Juntunen, K., and R. Halonen, "Self-care Portal as a Trigger for a Work Process Change: A Case from Finland", In Knowledge and Technologies in Innovative Information Systems. Springer, 2012, 225-240.

[17] Ketterer, T., D.W. West, V.P. Sanders, J. Hossain, M.C. Kondo, and I. Sharif, "Correlates of patient portal enrollment and activation in primary care pediatrics", Academic pediatrics 13(3), 2013, pp. 264-271.

[18] Kipping, S., M.I. Stuckey, A. Hernandez, T. 
Nguyen, and S. Riahi, "A web-based patient portal for mental health care: benefits evaluation", Journal of medical Internet research 18(11), 2016.

[19] Lyles, C.R., U. Sarkar, D. Schillinger, et al., "Refilling medications through an online patient portal: consistent improvements in adherence across racial/ethnic groups", Journal of the American Medical Informatics Association 23(e1), 2015, pp. e28-e33.

[20] Minniti, M.J., T.R. Blue, D. Freed, and S. Ballen, "Patient-interactive healthcare management, a model for achieving patient experience excellence", In Healthcare Information Management Systems. Springer, 2016, $257-$ 281.

[21] Mishuris, R.G., M. Stewart, G.M. Fix, et al., "Barriers to patient portal access among veterans receiving home-based primary care: a qualitative study", Health Expectations 18(6), 2015, pp. 2296-2305.

[22] Nambisan, P., "Factors that impact Patient Web Portal Readiness (PWPR) among the underserved", International journal of medical informatics 102, 2017, pp. 62-70.

[23] Noteboom, C.B., J. Hafner, and A. Wahbeh, "Characteristics of Complete and Incomplete Physicians' Unlearning with Electronic Medical Record", Journal of the Midwest Association for
Information Systems $\mid$ Vol 2017(2), 2017, pp. 57.

[24] Osborn, C.Y., L.S. Mayberry, K.A. Wallston, K.B. Johnson, and T.A. Elasy, "Understanding patient portal use: implications for medication management", Journal of medical Internet research 15(7), 2013.

[25] Palen, T.E., C. Ross, J.D. Powers, and S. Xu, "Association of online patient access to clinicians and medical records with use of clinical services", Jama 308(19), 2012.

[26] Peacock, S., A. Reddy, S.G. Leveille, et al., "Patient portals and personal health information online: perception, access, and use by US adults", Journal of the American Medical Informatics Association, 2016, pp. ocw095.

[27] Ralston, J.D., C.M. Rutter, D. Carrell, J. Hecht, D. Rubanowice, and G.E. Simon, "Patient use of secure electronic messaging within a shared medical record: a cross-sectional study", Journal of general internal medicine 24(3), 2009, pp. 349-355.

[28] Tenforde, M., A. Nowacki, A. Jain, and J. Hickner, "The association between personal health record use and diabetes quality measures", Journal of general internal medicine 27(4), 2012, pp. 420-424. 